\title{
Ville Korpela \\ Procedurally Fair Implementation: \\ The Cost of Insisting on Symmetry
}

\section{Aboa Centre for Economics}

Discussion paper No. 108

Turku 2016

The Aboa Centre for Economics is a joint initiative of the economics departments of the University of Turku and Åbo Akademi University.

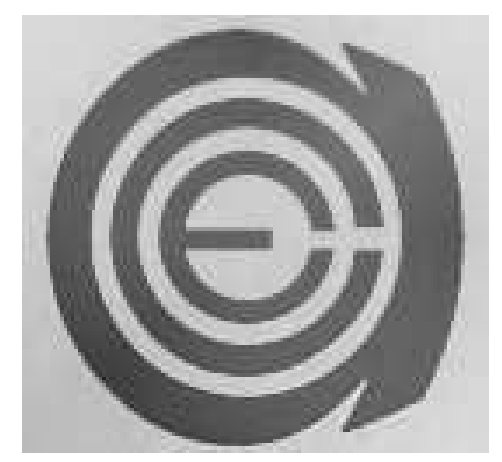


Copyright (C) Author(s)

ISSN 1796-3133

Printed in Uniprint Turku 2016 


\title{
Ville Korpela \\ Procedurally Fair Implementation: The Cost of Insisting on Symmetry
}

\author{
Aboa Centre for Economics \\ Discussion paper No. 108 \\ April 2016
}

\begin{abstract}
We derive a necessary and a sufficient condition for Nash implementation with a procedurally fair mechanism. Our result has a nice analogue with the path-braking result of Maskin [Nash equilibrium and welfare optimality, Rev. Econ. Stud. 66 (1999) 23-38.], and therefore, it allows us to give a simple characterization of those choice rules that are implementable, but not in a procedurally fair way. This reveals the constraints that insisting on procedural fairness impose on the collective.
\end{abstract}

JEL Classification: C72; D64; D70; D71

Keywords: Characterization; Implementation; Nash equilibrium; Other regarding preferences; Procedural fairness 
Contact information

Ville Korpela

Department of Economics

University of Turku

FI-20014, Finland

Email: ville.korpela (at) utu.fi

Acknowledgements

I thank the Academy of Finland for financial support. 


\section{Introduction}

Implementation theory studies the following question. There is a set of agents, a set of feasible outcomes, and a set of possible states that define preferences over these outcomes. A central agency, or the mechanism designer, wants to make a choice that depends on the state. The only thing that central agency can rely on in creating incentives is that preferences change between states. The issue is then whether a game, or a mechanism, can be designed so that at the equilibrium the wish of the central agency is fulfilled.

Although many theoretical questions have been solved by now, one central dilemma remains: Why are most mechanism in the real world so simple in comparison to the optimal mechanisms found in the theoretical literature. This is unfortunate since the focus of mechanism design has been on practical application from the beginning. The two leading explanations for this apparent simplicity are:

(1) That the mechanism design literature assumes too much common knowledge of the environment among the agents and the central agency. Therefore, a weakening of these assumption is needed to conduct useful analysis of practical problems. This is known as the Wilson doctrint $\oint^{1}$ and its implications are studied in Bergemann and Morris (2007) among others, and

(2) that the presence of competing mechanisms does not allow detailed fine tuning. This is well illustrated by the fact that the revelation principle does not necessarily hold when there is competition among mechanism designer (Peters and Epstein, 1999), but predicted already by the classic result of Rothschild and Stiglitz (1976), who show that there may not exist any equilibria in insurance markets since, given the insurance portfolio of one company, others can always reshape their own to attract more good customers 2

\footnotetext{
${ }^{1}$ Originally formulated by Wilson (1987).

${ }^{2}$ One could express this by saying that mechanism design should turn from partial
} 
Needless to say, frequently both, and many more for sure, have their own part to play. The goal, however, is to recognize the main driving force of simplicity. We study a third reason, and what kind of properties it implies for the choice rules, suggested by a casual observation:

(3) Often people do not care whether the goal of the central agency is fair or not, rather they simply demand that the decision making procedure must be fair [see Moulin (1997) for a general discussion]. This is most relevant in social choice problems, exemplified by the fact that most voting rules are fully symmetric, but can certainly have effects in other cases too.

There are many papers that investigate what can be implemented using a specific class of mechanism [Tian (1989), Sjöström (1994), Dutta, Sen, and Vohra (1995), Tatamitani (2001), Chen (2002), Mathevet (2010) 3 , but they do not give a general characterization of the constraints that insisting on procedural fairness impose, and then there are many papers that investigate exactly these constraints [Galbiati (2008), Rouillon (2013)], but only in specific settings. Therefore, a general characterization is obviously called for.

As a general rule the literature on fairness can be divided into two parts (Moulin, 1997): Papers that deal with procedural fairness (Azrieli and Jain, 2015) and papers that deal with end state fairness [Fleurbaey and Maniquet (1997), Sakai (2007)]. The difference is that procedural fairness is a property of the mechanism, while end state fairness is a property of the goal that central agency has. As already explained, our focus is on those cases where agents care only about the procedural fairness, and end state fairness plays no part. We strongly believe that this is the binding constraint in real wold applications.

The rest of the paper is organized as follows. In Section 2 we introduce the setup. In particular, we define what is meant by a procedurally fair mechanism. In Section 3 we define a property called permutation monotonicity, equilibrium analysis to general equilibrium analysis.

${ }^{3}$ For a general discussion see Saijo, Tatamitani, and Yamato (1996). 
which has a nice analogue with standard monotonicity, and show that it is a necessary condition for Nash implementation with a procedurally fair mechanism. Then, in Section 4, we show that permutation monotonicity and no-veto power are together sufficient for Nash implementation with a procedurally fair mechanism. Finally, Section 5 concludes. Here we explain how our characterization, together with that of Maskin (1999), can be seen as a characterization of the limitations that insisting on procedural fairness impose on the collective.

\section{The Setup}

Let $I=\{1, \ldots, n\}$ be a finite set of agents, $X$ a non-empty set of alternatives, and $\Theta$ the set of states. We assume throughout that the set of states is a subset of a product space i.e. $\Theta \subseteq \chi_{i=1}^{n} \Theta_{i}$. A choice rule (CR) $f: \Theta \rightarrow X$ associates a non-empty set of alternatives $f(\theta) \subseteq X$ to each state $\theta \in \Theta$. This represents the goal of a central agency (mechanism designer) and it is interpreted as selecting the optimal, or acceptable, alternatives at each state. However, central agency does not know the true state, only agents do. Therefore, it has to create incentives for the agents to reveal their information, and this can only be done if preferences change between states. The preference relation of agent $i$ at state $\theta \in \Theta$ is denoted by $\geq_{i}^{\theta}$.

Next we have to define what is the method that central agency can use to elicit the information from the agents. Given $n$ message spaces $M_{1}, \ldots, M_{n}$, a mechanism $g$ on $X$ is a mapping

$$
g: M_{1} \times \cdots \times M_{n} \rightarrow X
$$

We denote $M=M_{1} \times \cdots \times M_{n}$ and write this mechanism as $G=(M, g)$ t $^{4}$ Once a state $\theta \in \Theta$ is given, and preferences are therefore fixed, mechanism $G$ becomes a game $\Gamma(\theta)=(G, \theta)$. The message profile $m^{*}=\left(m_{1}^{*}, \ldots, m_{n}^{*}\right)$ is a pure strategy Nash equilibrium of this game if, and only if, $g\left(m^{*}\right) \geq_{i}^{\theta} g\left(m_{i}, m_{-i}^{*}\right)$

\footnotetext{
${ }^{4}$ Although $g$ already defines the message space.
} 
for all $i \in I$ and all $m_{i} \in M_{i} \stackrel{5}{5}^{6}$ The set of all pure strategy Nash equilibrium profiles of $\Gamma(\theta)$ is denoted by $N E(G, \theta)$. For arbitrary games the size of this set can be anything between empty and infinite.

We say that mechanism $G$ Nash implements $\operatorname{CR} f: \Theta \rightarrow X$ if $g(N E(G, \theta))=$ $f(\theta)$ for all $\theta \in \Theta$. In words, that is, if the outcomes at pure strategy Nash equilibrium profiles coincide exactly with the CR $f$. A given CR is then called Nash implementable if there exists some mechanism that Nash implements it. The path-braking result of Maskin (1999) says that if a CR is Nash implementable, then it is (Maskin) monotonic, and if it is monotonic and satisfies no-veto power (NVP), then it is Nash implementable. Choice rule $f$ is monotonic, if for all $\theta, \psi \in \Theta$, and all $x \in f(\theta)$, if $L_{i}(x, \theta) \subseteq L_{i}(x, \psi)$ for all $i \in I$, then $x \in f(\psi) .7$ It satisfies no-veto power, if for all $\theta \in \Theta$, and all $x \in X$, if $x$ is the best alternative of at least $n-1$ agents at state $\theta$, then $x \in f(\theta)$.

This result assumes, however, that central agency can use any mechanism it wants to. By contrast, we assume that the mechanism has to be procedurally fair. A permutation is a one-to-one function $\pi: I \rightarrow I$ and the set of all permutations is denoted by $\Pi$. When a permutation $\pi$ is applied to an $n$ -profile, for example to a state $\theta$, it means a permutation of the components, that is $\pi(\theta) \equiv\left(\theta_{\pi(1)}, \ldots, \theta_{\pi(n)}\right) \mathrm{S}^{8}$ In all our definition, given and forthcoming, we assume that either the set of alternatives $X$ does not depend on the identity of the agents, in which case we denote it by $A$, or it consists of $n$ -profiles, in which case we denote it by $Y$. The first case $X=A$ is a standard social choice setting, like a voting situation, and the second case $X=Y$ is a standard economic setting, like a resource allocation problem. We want to handle both cases at once.

Notice that in the first case permutation does not change the alternative,

\footnotetext{
${ }^{5}$ Here $m_{-i}^{*}=\left(m_{1}^{*}, \ldots, m_{i-1}^{*}, m_{i+1}^{*}, \ldots, m_{n}^{*}\right)$, and $\left(m_{i}, m_{-i}^{*}\right)=\left(m_{1}^{*}, \ldots, m_{i-1}^{*}, m_{i}, m_{i+1}^{*}, \ldots, m_{n}^{*}\right)$, as usual.

${ }^{6}$ We explain later on why it is legitimate to concentrate on pure strategies only.

${ }^{7}$ Monotonicity is called strong positive association by Muller and Satterthwaite (1977).

${ }^{8} \pi(\theta)$ is not necessarily in $\Theta$.
} 
that is $\pi(a)=a$ for all $(a, \pi) \in A \times \Pi$, and in the second case permutation affects the components of the alternative, that is $\pi(y)=\left(y_{\pi(1)}, \ldots, y_{\pi(n)}\right)$ for all $(y, \pi) \in Y \times \Pi$. Although it is reasonable to speak of state $\pi(\theta)$ even if it does not belong to $\Theta$, it is not reasonable to use $\pi(y)$ in any mechanism if it is not physically feasible i.e. $\pi(y) \notin Y$. Therefore, to avoid complications like this, we assume that $Y=\underset{i=1}{x} Z \mathfrak{\square}^{9}$

Definition 1. Mechanism $G=(M, g)$ is procedurally fair if for any permutation $\pi \in \Pi$, and any message $m \in M$, such that $g(m)=x$ and $g\left(M_{i}, m_{-i}\right)=X_{i}$ for all $i \in I$, there exists another message $m^{\prime} \in M$, such that $g\left(m^{\prime}\right)=\pi(x)$ and $g\left(M_{i}, m_{-i}^{\prime}\right)=\pi\left(X_{\pi(i)}\right)$ for all $i \in I$.

Here $X_{i}$ is the set of alternatives that agent $i$ can get by unilaterally deviating from $m$. If $X=A$, then $g\left(m^{\prime}\right)=\pi(x)$ transform to $g\left(m^{\prime}\right)=x$ and $\pi\left(X_{\pi(i)}\right)$ to $X_{\pi(i)}$, so the definition simplify considerably. The general condition $g\left(M_{i}, m_{-i}^{\prime}\right)=\pi\left(X_{\pi(i)}\right)$, which applies only in the case $X=Y$, means that agent $i$ is not only looking at the set $X_{\pi(i)}$ where agent $\pi(i)$ was able to deviate from $m$, but rather he is looking it placing himself in the shoes of agent $\pi(i)$.

Why do we not simply require full symmetry of the mechanism, that is $M_{1}=$ $M_{2}=\cdots=M_{n}$ and $g(\pi(m))=\pi(g(m))$ for all $(m, \pi) \in M \times \Pi$ ? The reason is that, as a representation of procedural fairness, this is too demanding. For example, suppose that $g(m)=x=\left(x_{1}, \ldots, x_{n}\right)$ and $m_{1}=m_{2}=\cdots=m_{n}$. Then, if we require full symmetry, it must be that

$$
\pi(x)=\pi(g(m))=g(\pi(m))=g(m)=x .
$$

As this holds for any permutation $\pi$, we get $x_{1}=x_{2}=\cdots=x_{n}$. However, there is nothing procedurally unfair in a mechanism where the agents can agree, by sending the same message, that an asymmetric outcome $x \in X$ should be selected. This is even more so if it is possible to agree on similar grounds that $\pi(x)$ should be selected by sending another message. The point is that full geometric symmetry is certainly not the same thing as

\footnotetext{
${ }^{9}$ Preferences can be other regarding since we do not assume that $x \geq y$ iff $x_{i} \geq y_{i}$.
} 
procedural fairness. The weaker assumption which says that if $g(m)=x$ for some $m \in M$, then there must exist another message profile $m^{\prime} \in M$, such that $g\left(m^{\prime}\right)=\pi(x)$, is part of our definition.

Definition 2. We say that CR $f: \Theta \rightarrow X$ is symmetric Nash implementable if, and only if, there exists a procedurally fair mechanism that Nash implements it.

In the rest of this paper we study what kind of properties procedural fairness, as we have defined it, implies for the choice rule 10

\section{Permutation Monotonicity}

The following property is to symmetric Nash implementation what monotonicity is to Nash implementation.

Definition 3. Choice rule $f: \Theta \rightarrow X$ is called permutation monotonic if for all $\theta, \psi \in \Theta$, all $x \in f(\theta)$, and all $\pi \in \Pi$, if $\pi\left(L_{\pi(i)}(x, \theta)\right) \subseteq L_{i}(\pi(x), \psi)$ for all $i \in I$, then $\pi(x) \in f(\psi)$.

If $X=A$, then this property is almost monotonicity, the only difference is that lower contour sets of $x$ at $\theta$ can be permuted in any way. That is, the condition on lower contour sets simplify to: $L_{\pi(i)}(x, \theta) \subseteq L_{i}(x, \psi)$ for all $i \in I$. On the other hand, if $X=Y$, then the condition is much more complicated since the set $\pi\left(L_{\pi(i)}(x, \theta)\right)$ is usually not the set $L_{\pi(i)}(\pi(x), \theta)$. This would not necessarily hold even if preferences are such that $x \geq_{i} y$ iff $x_{i} \geq_{i} y_{i}$.

Choice rule $f$ is called anonymous, if for all $\theta, \psi \in \Theta$, and all $\pi \in \Pi$, if $\geq_{i}^{\theta}=z_{\pi(i)}^{\psi}$ for all $i \in I$, then $f(\theta)=f(\psi)$ For an anonymous CR it does not matter who has which preferences. It is obvious that in the standard social choice setting permutation monotonicity implies both monotonicity (select $\pi=i d$ ) and anonymity (select $\pi$ in such way that $\geq_{i}^{\theta}=z_{\pi(i)}^{\psi}$ for all $i \in I$ ).

\footnotetext{
${ }^{10}$ See Pratt (2007) and Thomson (2011) for more on fairness.

${ }^{11}$ See Moulin (1988).
} 
However, the next example shows that monotonicity and anonymity are not together sufficient to imply permutation monotonicity even in this case.

Example 1. Let $I=\{1,2\}, A=\{a, b, c, d\}$, and $\Theta=\{\theta, \psi\}$. Preferences at different states are given in the table below ${ }^{12}$

State $\theta \quad$ State $\psi$

\begin{tabular}{cccc} 
Agent 1 & Agent 2 & Agent 1 & Agent 2 \\
\hline$c$ & $d$ & $b$ & $c$ \\
$a$ & $b$ & $d$ & $a$ \\
$b$ & $a$ & $a$ & $d$ \\
$d$ & $c$ & $c$ & $b$
\end{tabular}

Let the CR $f$ be such that $f(\theta)=\{a\}$ and $f(\psi)=\{d\}$. It is monotonic, since $L_{1}(a, \theta) \nsubseteq L_{1}(a, \psi)$ and $L_{1}(d, \psi) \nsubseteq L_{1}(d, \theta)$, and anonymous, since preferences at state $\theta$ are not a permutation of preferences at state $\psi$. However, it is not permutation monotonic since $L_{1}(a, \theta) \subseteq L_{2}(a, \psi)$ and $L_{2}(a, \theta) \subseteq L_{1}(a, \psi)$, while $a \notin f(\psi)$. $\diamond$

Our first theorem gives a necessary condition for a CR to be symmetric Nash implementable.

Theorem 1. If CR $f: \Theta \rightarrow A$ is symmetric Nash implementable, then it is permutation monotonic.

Proof. Suppose that $\theta, \psi \in \Theta, x \in f(\theta)$, and $\pi \in \Pi$, are such that $\pi\left(L_{\pi(i)}(x, \theta)\right) \subseteq L_{i}(\pi(x), \psi)$ for all $i \in I$. We have to show that $\pi(x) \in$ $f(\psi)$. Let $G=(M, g)$ be a procedurally fair mechanism that implements $f$. Since $x \in f(\theta)$, there must exists $m^{*} \in N E(G, \theta)$, such that $g\left(m^{*}\right)=x$, and $g\left(M_{i}, m_{-i}^{*}\right) \subseteq L_{i}(x, \theta)$ for all $i \in I$. Thus, by Definition 1 , there exists another message $m \in M$, such that $g(m)=\pi(x)$, and $g\left(M_{i}, m_{-i}\right) \subseteq \pi\left(L_{\pi(i)}(x, \theta)\right)$ for all $i \in I$. Therefore, by assumption, $m$ is a Nash equilibrium of $G$ at $\psi$. Finally, since $G$ implements $f$, this implies $\pi(x) \in f(\psi)$ as was to be shown.

This theorem make no assumptions about the number of agents. As a

\footnotetext{
${ }^{12}$ The convention here is that an alternative higher in the table is preferred.
} 
necessary condition, however, it can only give us negative conclusions.

Example 2 [Weak Pareto Correspondence]. An alternative $x \in X$ is weakly Pareto optimal at state $\theta$ if there does not exist another alternative $y$ such that $y>_{i}^{\theta} x$ for all $i \in I$. It is well-known that a CR which selects all weakly Pareto optimal alternatives at each states is Nash implementable if there are at least 3 agents (it is monotonic and satisfies NVP). However, all sub-correspondences of this CR are not Nash implementable, while some of them are ${ }^{13}$. Lets take another look at Example 1 where all alternatives $A=\{a, b, c, d\}$ are weakly Pareto optimal at both states.

Suppose that there is a third agent who has the same preference relation $a>b>c>d$ at both states $\theta$ and $\psi$. This guarantees that NVP is satisfied, and therefore implementability coincides with monotonicity. Furthermore, to check monotonicity, we do not need to worry about the agent with the same preference relation at both states. The $\mathrm{CR} f$ given in this example was monotonic, and hence Nash implementable, but not permutation monotonic, and therefore not by any procedurally fair mechanism. Let the CR $h$ be such that $h(\theta)=\{a\}$ and $h(\psi)=\{a, d\}$. It is easy to verify that this CR is permutation monotonic. In the next section we prove that $h$ is symmetric Nash implementable. $\diamond$

Example 3 [Individually Rational Correspondence]. Fix an alternative $x^{0} \in X$. Maskin (1999) defines the individually rational correspondence $f^{I R}$ : $\Theta \rightarrow X$ by the rule:

$$
f^{I R}\left(\theta ; x^{0}\right)=\left\{x \in X \mid x \geq_{i}^{\theta} x^{0} \text { for all } i \in I\right\} .
$$

Furthermore, he shows that the following mechanism Nash implements it: For each $i \in I$, let $M_{i}=X$ (the set of alternatives), and then define the outcome function $g^{I R}: M \rightarrow X$ as:

$$
g^{I R}(m)= \begin{cases}x, & \text { if } m_{1}=m_{2}=\cdots=m_{n}=x, \\ x^{0}, & \text { otherwise. }\end{cases}
$$

\footnotetext{
${ }^{13} g$ is a sub-correspondence of $f$ if $g(\theta) \subseteq f(\theta)$ for all $\theta \in \Theta$
} 
Is this mechanism procedurally fair? First of all, notice that the set $X_{i}$ in Definition 1 is always either $\{x\},\left\{x^{0}\right\}$ or $\left\{x, x^{0}\right\}$. If $X=A$, then for any

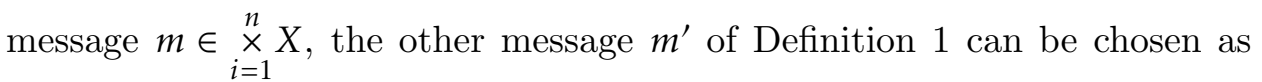
$m^{\prime}=\left(\pi\left(m_{1}\right), \pi\left(m_{2}\right), \ldots, \pi\left(m_{n}\right)\right)$, and therefore the answer is yes ${ }^{14}$ However, if $X=Y$, then it depends on how $x^{0}$ is selected. If this alternative is such that $x_{1}^{0}=x_{2}^{0}=\cdots=x_{n}^{0}$, then message $m^{\prime}$ can be chosen just as in the previous case. Therefore, $g^{I R}$ is procedurally fair in this special case, although not generally. By Theorem 1 this implies that $f^{I R}$ is permutation monotonic if $X=A$ or $X=Y$ and $x_{1}^{0}=x_{2}^{0}=\cdots=x_{n}^{0}$. $\diamond$

\section{Sufficient Conditions}

We are now ready to present the main result.

Theorem 2. Let $n \geq 3$. If CR $f: \Theta \rightarrow X$ is permutation monotonic and satisfies NVP, then it is symmetric Nash implementable.

Proof. To prove this claim we need a procedurally fair canonical mechanism. First of all, since state space $\Theta$ is not necessarily symmetric, we define a virtual state space $\Theta^{v}$ by the rule: For any $\pi \in \Pi$, and any $\theta \in \Theta$, let the state $\theta^{\pi}$ be such that the preference of agent $i$ is $\geq_{\pi(i)}^{\theta}$, and set

$$
\Theta^{v} \equiv\left\{\theta^{\pi} \mid \theta \in \Theta, \pi \in \Pi\right\}
$$

Notice that preferences at state $\theta^{\pi}$ and $\theta^{\pi^{\prime}}$ can be the same even if $\pi \neq$ $\pi^{\prime}$. Moreover, central agency knows that all states in $\Theta^{v}$ are not logically possible, it just needs to respect procedural fairness. Then, we expand $f$ into this set by defining a virtual choice rule (VCR) $f^{v}: \Theta^{v} \rightarrow X$ by the condition:

$$
f^{v}\left(\theta^{\pi}\right)=\pi(f(\theta)) \text { for all } \theta \in \Theta \text { and } \pi \in \Pi \text {. }
$$

This is the only way to expand $f$ in a symmetric way. The message space of agent $i$ is $M_{i}=\Theta \times \Pi \times X \times \mathbb{N}_{+}$, and a typical message of agent $i$ is denoted

\footnotetext{
${ }^{14}$ Notice that $\pi(m)$ is $\left(m_{\pi(1)}, m_{\pi(2)}, \ldots, m_{\pi(n)}\right)$ not $\left(\pi\left(m_{1}\right), \pi\left(m_{2}\right), \ldots, \pi\left(m_{n}\right)\right)$.
} 
by $m_{i}=\left(\theta^{i}, \pi^{i}, x^{i}, n^{i}\right)$. The outcome function $g: M \rightarrow X$ is then defined by the following rules:

(1) If $m_{i}=\left(\theta, \pi, x, n^{i}\right)$ for all $i \in I$, and $x \in f^{v}\left(\theta^{\pi}\right)$, then $g(m)=x$.

(2) If $m_{j}=\left(\theta, \pi, x, n^{j}\right)$ for all $j \in I \backslash\{i\}, m_{i}=\left(\theta^{i}, \pi^{i}, x^{i}, n^{i}\right)$, and $x \in f^{v}\left(\theta^{\pi}\right)$, then set 15

$$
g(m)= \begin{cases}x^{i}, & \text { if } x^{i} \in \pi\left(L_{i}\left(\pi^{-1}(x), \theta^{\pi}\right)\right), \\ x, & \text { otherwise. }\end{cases}
$$

(3) In all other cases, denote $k=\underset{i \in N}{\operatorname{argmax}} n^{i}$, and set

$$
g(m)=x^{k}
$$

REMARK: The fact that this mechanism has no mixed strategy equilibria can be proved exactly the same way as in Maskin (1999). Therefore, if integer games and infinite message spaces are allowed, then it is legitimate to focus on pure strategies only. $\diamond$

Lemma. This mechanism is procedurally fair.

proof. There are three cases to consider depending under which rule the outcome is calculated:

(1) Suppose that $m_{i}=\left(\theta, \pi, x, n^{i}\right)$ for all $i \in I, x \in f^{v}\left(\theta^{\pi}\right)$, and $g(m)=x$. Thus $g\left(M_{i}, m_{-i}\right)=\pi\left(L_{i}\left(\pi^{-1}(x), \theta^{\pi}\right)\right)$ for all $i \in I$. Let $\psi \in \Pi$. By Definition 1 we have to find a message profile $m^{\prime}$, such that $g\left(M_{i}, m_{-i}^{\prime}\right)=$ $\psi\left(\pi\left(L_{\psi(i)}\left(\pi^{-1}(x), \theta^{\pi}\right)\right)\right)$ for all $i \in I$, and $g\left(m^{\prime}\right)=\psi(x)$. Let $m^{\prime}$ be such that $m_{i}^{\prime}=\left(\theta, \psi \circ \pi, \psi(x), n^{i}\right)$ for all $i \in I\left[{ }^{16}\right.$ Then, since $\psi(x) \in f^{v}\left(\theta^{\psi \circ \pi}\right)$ by the definition of $f^{v}$, we have $g\left(m^{\prime}\right)=\psi(x)$, and moreover

$$
g\left(M_{i}, m_{i}^{\prime}\right)=\psi \circ \pi\left(L_{i}\left((\psi \circ \pi)^{-1}(\psi(x)), \theta^{\psi \circ \pi}\right)\right)=\psi\left(\pi\left(L_{\psi(i)}\left(\pi^{-1}(x), \theta^{\pi}\right)\right)\right)
$$

[since $(\psi \circ \pi)^{-1}=\pi^{-1} \circ \psi^{-1}$ for all $\pi, \psi \in \Pi$, we get $(\psi \circ \pi)^{-1}(\psi(x))=\pi^{-1}(x)$, and since $L_{i}\left(x, \theta^{\pi}\right)=L_{\pi(i)}(x, \theta)$ for all $(\theta, \pi) \in \Theta \times \Pi$, we get $\left.L_{i}\left(\pi^{-1}(x)\right), \theta^{\psi \circ \pi}\right)=$ $\left.\left.L_{\psi(i)}\left(\pi^{-1}(x)\right), \theta^{\pi}\right)\right]$.

\footnotetext{
${ }^{15}$ All permutations $\pi \in \Pi$ are invertible and $\pi^{-1} \in \Pi$.

${ }^{16} \psi \circ \pi$ is clearly a permutation.
} 
(2) Suppose that $m_{j}=\left(\theta, \pi, x, n^{j}\right)$ for all $j \in I \backslash\{i\}, m_{i}=\left(\theta^{i}, \pi^{i}, x^{i}, n^{i}\right)$, and $x \in f^{v}\left(\theta^{\pi}\right)$. Thus $g\left(M_{j}, m_{-j}\right)=X$ for all $j \in I \backslash\{i\}$ and $g\left(M_{i}, m_{-i}\right)=$ $\pi\left(L_{i}\left(\pi^{-1}(x), \theta^{\pi}\right)\right)$. Let $\psi \in \Pi$. By Definition 1 we have to find a message profile $m^{\prime}$, such that $g\left(M_{j}, m_{-j}^{\prime}\right)=X$ for all $\psi(j) \neq i, g\left(M_{\psi^{-1}(i)}, m_{-\psi^{-1}(i)}^{\prime}\right)=$ $\psi\left(\pi\left(L_{i}\left(\pi^{-1}(x), \theta^{\pi}\right)\right)\right)$, and $g\left(m^{\prime}\right)=\psi(x)$. Let $m^{\prime}$ be such that $m_{j}^{\prime}=(\theta, \psi \circ$ $\left.\pi, \psi(x), n^{i}\right)$ for all $\psi(j) \neq i$, and choose $m_{\psi^{-1}(i)}$ in such a way that the outcome is calculated using rule (2). Then, $g\left(M_{j}, m_{-j}\right)=X$ for all $\psi(j) \neq i$, and since $\psi(x) \in f^{v}\left(\theta^{\psi \circ \pi}\right)$ by the definition of $f^{v}$, we have $g\left(m^{\prime}\right)=\psi(x)$, and

$$
\begin{aligned}
& g\left(M_{\psi^{-1}(i)}, m_{-\psi^{-1}(i)}^{\prime}\right)=\psi \circ \pi\left(L_{\psi^{-1}(i)}\left((\psi \circ \pi)^{-1}(\psi(x)), \theta^{\psi \circ \pi}\right)\right)= \\
& \psi\left(\pi\left(L_{\psi\left(\psi^{-1}(i)\right)}\left(\pi^{-1}(x), \theta^{\pi}\right)\right)=\psi\left(\pi\left(L_{i}\left(\pi^{-1}(x), \theta^{\pi}\right)\right)\right)\right.
\end{aligned}
$$

[here we have used all those conditions that were used in the previous case as well with the additional fact that $\left.\psi\left(\psi^{-1}(i)\right)=i\right]$.

(3) This case is simple. Since $g\left(M_{i}, m_{-i}\right)=X$ for all $i \in I$, we can select $m^{\prime}=m$.

Let $G=(M, g)$ denote the mechanism defined above. We prove that it implements any CR that is monotonic and satisfies NVP and therefore it is canonical.

Suppose that $\theta \in \Theta$ is the true state, and $x \in f(\theta)$. Let the message profile $m=\left(m_{1}, \ldots, m_{n}\right)$ be such that $m_{i}=(\theta, i d, x, 1)$ for all $i \in N$. If agent $i$ deviates unilaterally to rule (2), he can get any alternative from the set $\pi\left(L_{i}\left(\pi^{-1}(x), \theta^{\pi}\right)\right)=L_{i}(x, \theta)$. Therefore, $m$ is a Nash equilibrium of $G$ at $\theta$, and since $g(m)=x$ by definition, we have shown that $f(\theta) \subseteq N E(G, \theta)$ for all $\theta \in \Theta$. A bit more work is need to prove the converse.

To begin with, if we have a Nash equilibrium where the outcome is calculated using rule (2) or (3), then, since the outcome must be the best alternative of at least $n-1$ agents in both cases, NVP guarantees that the outcome coincides with $f$. Suppose, therefore, that $m \in N E(G, \theta)$ and the outcome is calculated using rule (1). That is, for some $\psi \in \Theta$ and $y \in f^{v}\left(\psi^{\pi}\right)$, the message of agent $i \in N$ is $m_{i}=\left(\psi, \pi, y, n^{i}\right)$. Since $\psi^{\pi}$ is not necessarily the true state $\theta$, we need to show that $y \in f(\theta)$. 
By the definition of mechanism $G$, and the fact that $m \in N E(G, \theta)$, we get $\pi\left(L_{i}\left(\pi^{-1}(y), \psi^{\pi}\right)\right) \subseteq L_{i}(y, \theta)$. Then, since

$$
\pi\left(L_{i}\left(\pi^{-1}(y), \psi^{\pi}\right)\right)=\pi\left(L_{\pi(i)}\left(\pi^{-1}(y), \psi\right)\right),
$$

$\pi\left(\pi^{-1}(y)\right)=y$, and $\pi^{-1}(y) \in f(\psi)$ by definition, permutation monotonicity implies $y \in f(\theta)$ as was to be shown. This complete the proof.

This theorem has a nice analogue with the corresponding result of Maskin (1999) given in Sect. 2 - it is just that monotonicity is replaced with permutation monotonicity.

Example 4 (No-Envy Correspondence). There is a bundle of goods $\Omega \in \mathbb{R}_{++}^{l}$ to be distributed. Let $z_{i} \in \mathbb{R}_{+}^{l}$ be the bundle that is given to agent $i, Z=\left\{z \in \mathbb{R}_{+}^{l n} \mid \sum_{i=1}^{n} z_{i}=\Omega\right\}$ the set of feasible allocations, and $Z_{0}=\left\{z^{0} \in\right.$ $\left.\mathbb{R}_{+}^{n} \mid z^{0} \leq \Omega\right\}$ the set of possible consumption bundles for any agent 17

At each state $\theta \in \Theta$, the preference relation $\geq_{i}^{\theta}$ of agent $i$ is continuous, convex, and strictly monotone over $\mathbb{R}_{+}^{l}$. Now we assume, as usual, that $x \geq_{i}^{\theta} y$ iff $x_{i} \geq_{i}^{\theta} y_{i}$. An allocation $z \in Z$ is called envy-free at state $\theta$ if $z_{i} \geq_{i}^{\theta} z_{j}$ for all pairs of agents $i, j \in I$ In In words, no agent $i$ prefers the bundle of another agent $z_{j}$ to his own $z_{i}$. The no-envy correspondence is then defined as a choice rule ef $: \Theta \rightarrow Z$ that selects all envy-free allocations at each state.

This correspondence satisfies NVP if there are at least three agents since the best allocation is to get the entire endowment $\Omega$ and leave everyone else with nothing. Thomson (2005) gives a nice mechanism, that he calls the "divide and permute" -mechanism, which Nash implements ef. Therefore, by the theorem of Maskin (1999), it must be monotonic as well. It is not, however, procedurally fair. Nevertheless, from the work of Galbiati (2008), we known that ef can be Nash implemented with a procedurally fair mechanism. By Theorem 2 this means that ef must be permutation monotonic. We verify this directly.

\footnotetext{
${ }^{17} z \in \mathbb{R}_{+}^{l}$ means that all components of $z$ are non-negative and $z \in \mathbb{R}_{++}^{l n}$ means that all components of $z$ are positive. Moreover, $z^{0} \leq \Omega$ means that $z_{i}^{0} \leq \Omega_{i}$ for all $i \in I$.

${ }^{18}$ This concept was originally defined in Foley (1967).
} 
Suppose that $\theta, \psi \in \Theta, x \in e f(\theta)$, and $\pi \in \Pi$, are such that $\pi\left(L_{\pi(i)}(x, \theta)\right) \subseteq$ $L_{i}(\pi(x), \psi)$ for all $i \in I$. Assume that $\pi(x) \notin e f(\psi)$. By definition this means that $\pi(x)_{j}>_{i}^{\psi} \pi(x)_{i}$ for some $i, j \in I$, or equivalently, that $x_{\pi(j)}>_{i}^{\psi} x_{\pi(i)}$ for some $i, j \in I$. Hence $x_{\pi(j)}>_{i}^{\psi} y_{i}$ for all $y \in L_{i}(\pi(x), \psi)$, and therefore $x_{\pi(j)} \neq z_{\pi(j)}$ must hold for all $z \in \pi\left(L_{\pi(i)}(x, \theta)\right)$ by assumption. This means that $x_{j} \neq z_{j}$ for all $z \in L_{\pi(i)}(x, \theta)$, which implies $x_{j}>_{\pi(i)}^{\theta} x_{\pi(i)}$, so that $x \notin e f(\theta)$ - a contradiction. Therefore $\pi(x) \in e f(\psi)$ and ef is indeed permutation monotonic.

Just like in the case of weak Pareto correspondence, a sub-correspondence of ef is not necessarily monotonic, and therefore not necessarily permutation monotonic either, so this has to be checked every time.

\section{Concluding Discussion}

We have derived a necessary [Theorem 1] and a sufficient [Theorem 2] condition for a CR to be Nash implementable by a procedurally fair mechanism or symmetric Nash implementable. Although our characterization is simple and it has a nice intuitive interpretation, which is a desirable feature for this type of results, the most interesting result comes as a corollary from a comparison to the corresponding result of Maskin (1999). Recall that no-veto power holds vacuously in any resource allocation problem (see Example 4).

Corollary. Choice rule is Nash implementable in an economic environment, but not by any procedurally fair mechanism if, and only if, it is monotonic, but not permutation monotonic.

We saw in Example 2 that if a CR is efficient in the sense of weak Pareto optimality, which is a minimal desideratum in economic environments, then the constraints imposed by permutation monotonicity are stronger than those imposed by standard monotonicity.

Also another interesting observation emerge from a comparison to existing literature. In a recent paper Azrieli and Jain (2015) study the same problem 
as we do here, except that they focus on the case of incomplete information, and hence use Bayes-Nash equilibrium as the solution concept. This is not the only difference, however, since they rely on the revelation principle, and therefore study partial implementation rather than full implementation as we do here. Interestingly, although not surprisingly, their result stands in a stark contrast to ours. While our conclusion is that symmetry (procedural fairness) is a strong constraint on implementability, since permutation monotonicity is a lot more demanding than monotonicity, their conclusion is that symmetry is only a weak constraint 19

There is a simple explanation for this apparent contradiction however. In full implementation symmetry implies that with one equilibrium a symmetric equilibrium is created alongside, while in partial implementation, with focus on the truthful Bayes-Nash equilibrium only, this other equilibrium can be neglected. Azrieli and Jain (2015) explain this by observing that symmetry is not severely binding since the truthful equilibrium can be asymmetric. This is not possible in full implementation since also the symmetric equilibrium has to be accounted for.

\footnotetext{
${ }^{19}$ The contrast is made even deeper by the fact that their concept of symmetry is stronger than ours.
} 


\section{References}

Azrieli, Y. and Jain, R. (2015): Symmetric mechanism design. Working Paper, Unpublished.

Bergemann, D. and Morris, S. (2007): "Robust mechanism design". Econometrica 73(6): 1771-1813.

Chen, Y. (2002): "A family of supermodular Nash mechanisms implementing Lindahl allocations". Economic Theory 19(4): 773-790.

Dutta, B., Sen, A. and Vohra, R. (1995): "Nash implementation through elementary mechanisms in economic environments". Economic Design 1: 173-204.

Fleurbaey, M. and Maniquet, F. (1997): "Implementability and horizontal equity imply no-envy". Econometrica 65: 1215-1219.

Foley, D. (1967): "Resource allocation and the public sector". Yale Economic Essays 7: 45-98.

Galbiati, M. (2008): "Fair divisions as attracting Nash equilibria of simple games". Economics Letters 100(1): 72-75.

Maskin, E. (1999): "Nash equilibrium and welfare optimality". Review of Economic Studies 66: 23-38.

Mathevet, L. (2010): "Supermodular Mechanism Design". Theoretical Economics 5(3): 403-443.

Moore, J. and Repullo, R. (1990): "Nash implementation: a full characterization". Econometrica 58: 1083-1099.

Moulin, H. (1997): Procedural cum Endstate Justice: An Implementation Viewpoint. Duke University Working Papers, Department of Economics.

Moulin, H. (1988): Axioms of Cooperative Decision Making. Cambridge University Press, USA.

Muller, E. and Satterthwaite, M. (1977): "The Equivalence of Strong Positive Association and Strategy-Proofness". Journal of Economic Theory 14: 412-418.

Peters, M. and Epstein, L. (1999): "A Revelation Principle for Competing Mechanisms". Journal of Economic Theory 88: 119-160.

Pratt, J. W. (2007): "Fair (and not so fair) division". Journal of Risk and 
Uncertainty 35: 203-236.

Rouillon, S. (2013): "Anonymous implementation of the Lindahl correspondence: possibility and impossibility results". Social Choice and Welfare 40: 1179-1203.

Rothschild, M. and Stiglitz, J. (1976): "Equilibrium in Competitive Insurance Markets: An Essay on the Economics of Imperfect Information". The Quarterly Journal of Economics 90(4): 629-649.

Saijo, T., Tatamitani, Y. and Yamato, T. (1996): "Toward natural implementation". International Economic Review 37: 949-980.

Sakai, T. (2007): "Fairness and implementability in allocation of indivisible objects with monetary compensations". Journal of Mathematical Economics 43(5): 549-563.

Sjöström, T. (1994): "Implementation by Demand Mechanisms". Economic Design 1(1): 343-354.

Tatamitani, Y. (2001): "Implementation by Self-Relevant Mechanisms". Journal of Mathematical Economics 35(3): 427-444.

Thomson, W. (2011): Fair Allocation Rules. In Handbook of Social Choice and Welfare Vol. 2, ed. by K. J. Arrow, A. K. Sen and K. Suzumura, Elsevier.

Thomson, W. (2005): "Divide-and-permute". Games and Economic Behavior 52(1): 186-200.

Tian, G. (1989): "Implementation of the Lindahl correspondence by a single-valued, feasible and continuous correspondence". The Review of Economic Studies 56: 613-621.

Wilson, R. (1987): "Game-Theoretic Analyses of Trading Processes". Chap. 2 in Advances in Economic Theory: Fifth World Congress, ed. by T. Bewley. Cambridge, U.K., Cambridge University Press: 33-70. 
The Aboa Centre for Economics (ACE) is a joint initiative of the economics departments of the Turku School of Economics at the University of Turku and the School of Business and Economics at Åbo Akademi University. ACE was founded in 1998. The aim of the Centre is to coordinate research and education related to economics.

Contact information: Aboa Centre for Economics, Department of Economics, Rehtorinpellonkatu 3, FI-20500 Turku, Finland.

www.ace-economics.fi

ISSN 1796-3133 